University of Nebraska - Lincoln

DigitalCommons@University of Nebraska - Lincoln

Faculty Publications from the Department of Electrical \& Computer Engineering, Department Electrical and Computer Engineering

$11-1-2000$

\title{
Dielectric function of amorphous tantalum oxide from the far infrared to the deep ultraviolet spectral region measured by spectroscopic ellipsometry
}

\author{
Eva Franke \\ University of Nebraska-Lincoln, efranke3@unl.edu \\ C. L. Trimble \\ University of Nebraska-Lincoln \\ M. J. DeVries \\ University of Nebraska-Lincoln \\ John A. Woollam \\ University of Nebraska-Lincoln, jwoollam1@unl.edu \\ Mathias Schubert \\ University of Nebraska-Lincoln, mschubert4@unl.edu \\ See next page for additional authors \\ Follow this and additional works at: https://digitalcommons.unl.edu/electricalengineeringfacpub \\ Part of the Electrical and Computer Engineering Commons
}

Franke, Eva; Trimble, C. L.; DeVries, M. J.; Woollam, John A.; Schubert, Mathias; and Frost, F., "Dielectric function of amorphous tantalum oxide from the far infrared to the deep ultraviolet spectral region measured by spectroscopic ellipsometry" (2000). Faculty Publications from the Department of Electrical and Computer Engineering. 17.

https://digitalcommons.unl.edu/electricalengineeringfacpub/17

This Article is brought to you for free and open access by the Electrical \& Computer Engineering, Department of at DigitalCommons@University of Nebraska - Lincoln. It has been accepted for inclusion in Faculty Publications from the Department of Electrical and Computer Engineering by an authorized administrator of DigitalCommons@University of Nebraska - Lincoln. 


\section{Authors}

Eva Franke, C. L. Trimble, M. J. DeVries, John A. Woollam, Mathias Schubert, and F. Frost 


\title{
Dielectric function of amorphous tantalum oxide from the far infrared to the deep ultraviolet spectral region measured by spectroscopic ellipsometry
}

\author{
Eva Franke, ${ }^{\text {a) }}$ C. L. Trimble, M. J. DeVries, and J. A. Woollam \\ Center for Microelectronic and Optical Materials Research and Department of Electrical Engineering, \\ University of Nebraska, Lincoln, Nebraska 68588-0511 \\ M. Schubert \\ Center for Microelectronic and Optical Materials Research and Department of Electrical Engineering, \\ University of Nebraska, Lincoln, Nebraska 68588-0551 and Faculty of Physics and Geoscience, \\ Solid State Optics and Semiconductor Physics Group, Linnéstrasse 5, D-04103 Leipzig, Germany \\ F. Frost \\ Institut für Oberflächenmodifizierung Leipzig e.V., Permoserstr. 15, 04318 Leipzig, Germany
}

(Received 21 January 2000; accepted for publication 3 August 2000)

\begin{abstract}
Amorphous tantalum oxide thin films were deposited by reactive rf magnetron sputtering onto [001] silicon substrates. Growth temperature, oxygen partial pressure, and total gas pressure have been varied to obtain thin films with different densities. The thin films were analyzed by glancing angle-of-incidence x-ray diffraction, atomic force microscopy, and variable angle-of-incidence spectroscopic ellipsometry in the near infrared to vacuum ultraviolet spectral region for photon energies from $E=1$ to $8.5 \mathrm{eV}$, and in the infrared region from $E=0.03$ to $1 \mathrm{eV}$. We present the dielectric function of amorphous tantalum oxide obtained by line shape analysis of the experimental ellipsometric data over the range from $E=0.03$ to $8.5 \mathrm{eV}(40 \mu \mathrm{m}-145 \mathrm{~nm})$. In the infrared spectral region the ellipsometric data were analyzed using Lorentzian line shapes for each absorption mode observed in the spectra. Amorphous tantalum oxide optical properties in the near infrared to vacuum ultraviolet spectral region were extracted by using a Kim and Garland parameter algorithm [C. C. Kim et al., Phys. Rev. B 45, 11749 (1992)] in order to model the absorption due to the fundamental band gap of the material. We consider thin film porosity, and therefore analyzed the experimental ellipsometric data by an effective medium approach. We obtain information on the tantalum oxide optical properties, a percentage of void fraction, and film thickness. The "optical" percentage of void fractions corresponds to surface roughness measured by atomic force microscopy and depends on deposition parameters. (C) 2000 American Institute of Physics. [S0021-8979(00)05821-7]
\end{abstract}

\section{INTRODUCTION}

Sputtered thin-film materials are widely used for optical applications in high-quality antireflection coatings or optical filters. Tantalum oxide has large refractive index values, and low extinction coefficients over a wide spectral range. Tantalum oxide thin films are therefore of high interest for optical devices, and can be used as a high refractive index material in multilayer interference filters, ${ }^{1}$ as antireflection coatings for solar cells, ${ }^{1,2}$ or in optical wave guides. ${ }^{3}$ Thin films of tantalum oxide are also employed as an ion conductor in electrochromic devices. ${ }^{4,5}$ Known as a material with high static dielectric constant and with good electrical insulating properties, tantalum oxide has the potential for microelectronic applications as a capacitor and gate material, thereby replacing silicon dioxide in metal-insulator-metal ${ }^{6-11}$ or metal-insulator-semiconductor $^{6-10,12-17}$ structures as well as in large-scale integrated circuits for dynamic random access memories. ${ }^{6}$

Sputter-deposited thin films tend to show film porosity, which affects electrical or optical properties. Sample aging

a)Electronic mail: franke@engrs.unl.edu due to moisture incorporation followed by chemical degradation may decrease the electrical resistance, and increase light scattering at rough interfaces. The film porosity, i.e., the film microstructure, depends mostly on growth conditions. Prior to application of sputter-deposited films it is necessary to understand the relation between microstructure, growth conditions, and resulting electrical and optical properties. A through optical study of such microstructure related effects was shown previously for magnetron sputtered boron nitride thin films ${ }^{18-21}$

Tantalum oxide films (TOF) have been grown by a large variety of physical vapor deposition and chemical vapor deposition (CVD) processes, including ion beam sputtering, ${ }^{22,23}$ magnetron sputtering, ${ }^{2,7,9,24}$ (low temperature) thermal oxidation, ${ }^{9,25}$ anodization, ${ }^{26}$ ion plating, ${ }^{1,27}$ electronbeam evaporation, ${ }^{4,27}$ laser ablation, ${ }^{28,29}$ low pressure CVD,${ }^{14,30}$ photo-CVD, ${ }^{31,32}$ atomic layer deposition, ${ }^{33}$ and plasma-enhanced CVD. ${ }^{12,16,34}$ A metalorganic solution deposition technique ${ }^{6}$ was also used to grow TOF. Low temperature grown TOFs tend to be amorphous. Postannealing of the amorphous samples at elevated temperatures above $700{ }^{\circ} \mathrm{C}$ causes crystallization. ${ }^{16}$ Depending on the annealing history, 
TABLE I. Band-to-band transition energies obtained from tantalum oxide thin films grown by different deposition techniques (am=amorphous, pol $=$ polycrystalline, refl $=$ reflectance, $\operatorname{tran}=$ transmission $)$

\begin{tabular}{|c|c|c|c|c|}
\hline Growth technique & Phase & Optical method & $\begin{array}{c}\text { Band gap } \\
{[\mathrm{eV}]}\end{array}$ & Remarks \\
\hline reactive sputtering & & refl, and tran & $\begin{array}{c}4.07\left(E_{g 1}\right)^{\mathrm{a})} \\
4.5\left(E_{g_{2}}\right)\end{array}$ & \\
\hline electron-beam evaporation & & $\operatorname{tran}$ & $\begin{array}{l}4.53\left(E_{g 1}\right)^{\mathrm{b})} \\
4.71\left(E_{g 1}\right)\end{array}$ & $\begin{array}{l}\text { TOF grown at } 100^{\circ} \mathrm{C} \\
\text { TOF grown at } 200^{\circ} \mathrm{C}\end{array}$ \\
\hline ion-beam sputtering & am & $\operatorname{tran}$ & $\begin{array}{l}\left.4.0\left(E_{g 1}\right)^{\mathrm{c}}\right) \\
4.3\left(E_{g 2}\right)\end{array}$ & $\begin{array}{l}\text { substoichiometric } \\
\text { stoichiometric }\end{array}$ \\
\hline metalorganic solution technique & pol & refl-ellipsometry & $5.11^{\mathrm{d})}$ & \\
\hline photoinduced CVD & pol & $\operatorname{tran}$ & $4.2^{\mathrm{e})}$ & \\
\hline atomic layer deposition & $\begin{array}{c}\text { am and } \\
\text { pol }\end{array}$ & $\operatorname{tran}$ & $\begin{array}{c}4.0\left(E_{g 1}\right)^{\mathrm{f})} \\
4.2\left(E_{g 1}\right) \\
3.9 \ldots . .5\end{array}$ & $\begin{array}{l}\text { substoichiometric } \\
\text { amorphous } \\
\text { polycrystalline }\end{array}$ \\
\hline magnetron sputtering & am & refl-ellipsometry & $\begin{array}{c}4.62 \ldots 4.88\left(E_{g 1}\right)^{\mathrm{g})} \\
5.44 \ldots 5.52\left(E_{g_{2}}\right)\end{array}$ & \\
\hline
\end{tabular}

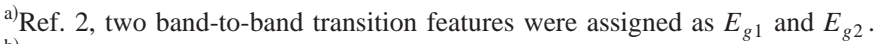

${ }^{b}$ Ref. 4 , two films grown at different substrate temperatures were studied.

${ }^{\text {c) }}$ Ref. 22, the features at $E_{g 1}$ and $E_{g 2}$ were assigned to substoichiometric and stoichiometric phases, respectively.

${ }^{d)}$ Ref. 6, band-gap value was assigned to crystalline TOF.

${ }^{\text {e) }}$ Ref. 31 .

${ }^{\text {f) }}$ Ref. 33, a large range of band-to-band transitions was found for polycrystalline TOFs.

${ }^{g}$ Present work.

either orthorhombic ${ }^{6,8,15,29}$ or hexagonal ${ }^{14}$ phases can be obtained.

Earlier TOF studies concentrated on electrical properties, such as the static dielectric constant and the leakage current across the films. Many reports also focused on TOF optical properties in the near infrared (NIR)-UV spectral region from 1 to $5 \mathrm{eV}$.,2,12,22,23,27,31,33,35 Transmission and reflectance data were analyzed to study the dispersion of index of refraction $n$ and extinction coefficient $k$, and position of the fundamental band-gap transition. Rubio et al. ${ }^{2}$ reported a strong increase of $n$ and $k$ for short wavelengths $\lambda(\lambda<0.4 \mu \mathrm{m})$, and assigned a phonon-assisted indirect electronic transition at $E_{g}=4.07 \mathrm{eV}$. A second band-to-band transition feature was found at $E_{g}=4.5 \mathrm{eV}$. Demiryont et $a l .{ }^{23}$ investigated optical properties of TOFs with different stoichiometries. The authors found that an increasing oxygen deficiency is related to higher $n$ and $k$ values. This is particularly evident in $k$ where the extinction coefficient is almost zero below the band gap for stoichiometric films and raises to values close to 0.1 for cermet films (TOF with metallic Ta cluster). The onset of absorption in $n$ and $k$ due to the band gap within the stoichiometric film is rather sharp, whereas the substoichiometric and cermet films show a smoother dispersion with increasing oxygen deficiency. The position of the fundamental band gap is reported to be slightly composition dependent: $E_{g}=4.3 \mathrm{eV}$ for stoichiometric and $E_{g}$ $=4.0 \mathrm{eV}$ for substoichiometric TOFs. The band gap in cermet films depends on the cluster concentration, and varies from $E_{g}=3 \mathrm{eV}$ to almost metallic behavior. The influence of stoichiometry on the optical properties was also studied in Refs. 12 and 22. Therein the authors also report increased $n$ values for oxygen deficient TOFs. The refractive index $n$ was also shown to be a function of the deposition temperature, ${ }^{12,31,33}$ and total gas pressure ${ }^{24,31}$ during sample growth. Because temperature and total gas pressure affect the thin-film porosity, ${ }^{24}$ the TOF refractive index also increases by thin-film densification. Hence, an effective refractive index of TOF results from growth conditions, and may range from $n=2.06$ (evaporation) $^{27}$ to $n=2.25$ (ion plating) ${ }^{27}$ at $\lambda=550 \mathrm{~nm}$. The differences in $n$ are mostly due to different thin-film porosity, ${ }^{22,27}$ which is known to be higher for films grown by evaporation than by ion plating. It was also shown that mixtures of substoichiometric and stoichiometric tantalum oxide causes lower $n$ values in TOFs. ${ }^{27}$

Porqueras et al. ${ }^{4}$ and Kukli et al. ${ }^{33}$ studied the fundamental band-to-band transition energy as a function of growth temperature. TOFs investigated by Porqueras et al. ${ }^{4}$ were highly transparent below the band gap, which was reported as a direct electronic band-to-band transition. The square of the absorption coefficient $\alpha=2 \pi k / \lambda$ versus photon energy $E$ was found to increase almost linearly with $E$. Band-gap values of 4.53 and $4.71 \mathrm{eV}$ were reported for films grown at 100 and $200{ }^{\circ} \mathrm{C}$, respectively. ${ }^{4}$ In contrast to Porqueras et $a l .{ }^{4}$ Kukli et al. ${ }^{33}$ observed a $\left(E-E_{g}\right)^{2}$ dependence for the absorption coefficient in TOFs and concluded an indirect electronic band-to-band transition mechanism. Mixedphase TOFs grown at higher temperatures reveal two bandto-band transition features at $E_{g 1}=4.0 \mathrm{eV}$ and $E_{g 2}=4.5 \mathrm{eV}^{33}$ The origin of $E_{g 1}$ and $E_{g 2}$ was assigned to contributions of $\mathrm{TaO}_{2}$ and $\mathrm{Ta}_{2} \mathrm{O}_{5}$, respectively. A summary of the band-gap values obtained for TOFs deposited by various growth techniques is given in Table I.

A band-gap value of $5.11 \mathrm{eV}$ has been obtained for CVD-grown polycrystalline TOFs. ${ }^{6}$ In spite of the results by Joshi and Cole ${ }^{6}$ it was claimed that a phase transition from amorphous to crystalline causes a decrease in band gap. ${ }^{33}$

No theoretical studies are reported for the band structure of amorphous tantalum oxide. The available experimental results for the lowest band-to-band transition mechanism are contradictory. It is very likely, that the absorption properties 
of TOFs will depend on their phase composition and stoichiometry.

To the best of our knowledge no data exist for the optical constants $(n, k)$ or the dielectric functions $\left(\epsilon_{1}, \epsilon_{2}\right)$ of amorphous and crystalline tantalum oxide in the IR spectral region. IR transmission intensity spectra from $\omega=400$ to $4000 \mathrm{~cm}^{-1}$ of amorphous and crystalline TOFs are shown in Refs. 13 and 29-31. No line-shape analysis of the IR spectra to extract the dielectric function was performed. A strong absorption feature near $\omega=635 \mathrm{~cm}^{-1}$ was assigned to the transverse optical (TO) phonon of amorphous tantalum oxide. $^{13,29}$ After sample annealing, the absorption peak moved to lower frequencies near $\omega=520 \mathrm{~cm}^{-1}$ possibly due to crystallization of the films. ${ }^{13,29}$ Substoichiometric TOFs reveal a broad absorption band between $\omega=700$ and 1100 $\mathrm{cm}^{-1} \cdot{ }^{29,31}$ Moisture adsorption in as-grown TOFs was detected with characteristic $\mathrm{H}_{2} \mathrm{O}$ absorption lines within the IR spectra. ${ }^{31}$ No published optical data for tantalum oxide are available for photon energies from 5.5 to $8.5 \mathrm{eV}$.

In this work we study the optical properties of amorphous tantalum oxide thin films grown by rf magnetron sputtering on [001] silicon. Growth temperature, oxygen flux, and total gas pressure was varied, and films with different microporosities were obtained. For measurement of the TOF optical properties we utilize spectroscopic ellipsometry for photon energies from 0.03 to $8.5 \mathrm{eV}$. The ellipsometry data are analyzed with parametric dielectric function models throughout the spectral region investigated. An effective medium approach ${ }^{36}$ is employed to explore the optical effect of the thin-film porosity, which was studied by assuming that all micropores are filled by adsorbed water content (moisture fraction). As a result we present the optical dielectric function spectra of amorphous tantalum oxide for photon energies from 0.03 to $8.5 \mathrm{eV}$ (wavelengths from $40 \mu \mathrm{m}$ to 145 $\mathrm{nm})$.

The TOFs microstructure was studied by glancing angleof-incidence X-ray diffraction (XRD) investigations, and atomic force microscopy (AFM). Results for surface roughness obtained by ellipsometry and AFM measurements are compared to each other.

\section{EXPERIMENT}

Amorphous TOFs were grown by reactive $\mathrm{rf}$ magnetron sputtering onto [001] $\mathrm{Si}$ substrates in a high vacuum chamber with a basis pressure of $1 \times 10^{-6}$ Torr. A metal tantalum target was sputtered using an argon-oxygen mixture of $22 \%$ or $40 \%$ oxygen content. rf power was held constant at $P$ $=100 \mathrm{~W}$ during deposition of all samples. Oxygen flux $f_{\mathrm{O} 2}$, growth temperature, and total pressure $p_{\text {tot }}$ were varied to obtain different film microporosities. Sample deposition parameters are summarized in Table II.

Glancing angle-of-incidence XRD measurements were performed in order to obtain the TOF microstructure and phase compositions. The measurements were taken with a commercial diffractometer using $\mathrm{Cu} K_{\alpha}$ radiation. The diffraction angle was scanned between $20^{\circ} \leqslant 2 \theta \leqslant 60^{\circ}$. Diffraction peaks related to crystalline phases of tantalum oxide are expected within this region. ${ }^{7}$
TABLE II. Sample growth parameters used in this work. Argon flux was 20 sccm for all samples, and rf power was $100 \mathrm{~W}$.

\begin{tabular}{cccc}
\hline \hline Sample & $\begin{array}{c}\text { Temperature } \\
{\left[{ }^{\circ} \mathrm{C}\right]}\end{array}$ & $f_{\text {O2 }}[\mathrm{sccm}]$ & {$\left[p_{\text {tot }}\right][\mathrm{mTorr}]$} \\
\hline Ta1 & 20 & 4.5 & 15 \\
Ta2 & 20 & 8.0 & 15 \\
Ta3 & 100 & 4.5 & 15 \\
Ta4 & 200 & 4.5 & 15 \\
Ta5 & 20 & 4.5 & 17 \\
\hline \hline
\end{tabular}

Surface scans were performed in contact mode using a commercial AFM instrument in order to study morphology of the TOFs. We register the root mean square ( $\mathrm{rms}$ ) value (standard deviation of the measured height values, i.e., the standard deviation of the elongation of the AFM cantilever tip) as a measure of the surface roughness. The rms value was determined for sample surface scan ranges of $2 \mu \mathrm{m}$.

All samples were measured at room temperature using commercial variable angle-of-incidence spectroscopic ellipsometers (SE) for photon energies $E$ from 0.03 to $8.5 \mathrm{eV}$. A rotating-analyzer ellipsometer equipped with a $75 \mathrm{~W}$ xenon lamp as a light source was employed for the spectral region from 0.75 to $5.5 \mathrm{eV}$, i.e., from the NIR to the UV. Measurements were taken at multiple angles of incidence $\Phi_{a}=65^{\circ}$, $70^{\circ}, 75^{\circ}$, and $80^{\circ}$. The NIR-UV ellipsometer system is further equipped with an automated compensator which allows accurate determination of ellipsometric parameter $\Delta$.

Spectroscopic ellipsometry experiments within the IR (IR-SE) were carried out in the wave number range from 300 to $8000 \mathrm{~cm}^{-1}(0.03-1 \mathrm{eV})$ at one angle of incidence $\Phi_{a}=65^{\circ}$. A commercial rotating-polarizer, rotatingcompensator, Fourier-transform based variable angle-ofincidence ellipsometer was used.

For measurements in the vacuum-UV (VUV) spectral region (5-8.5 eV, wavelengths $248-145 \mathrm{~nm}$ ) the samples were placed in a chamber purged with dry nitrogen. A rotating-analyzer ellipsometer with automated VUV compensator, and VUV polarizers was attached to the chamber (VUV-SE). A deuterium lamp was used as the light source. Measurements were taken at a single angle of incidence of $\Phi_{a}=75^{\circ}$.

\section{THEORY}

Ellipsometry can be used to determine the dielectric function $\epsilon$ and thickness of thin films by comparing the measured data with a model calculation. The standard ellipsometric parameters are defined by $\Psi$ and $\Delta$. They are related to the complex reflectance ratio $\rho$ with

$$
\rho=\frac{R_{p}}{R_{s}}=\tan \Psi \exp (i \Delta),
$$

where $R_{p}$ and $R_{s}$ are the reflection coefficients for light polarized parallel $(p)$ and perpendicular $(s)$ to the plane of incidence, respectively. ${ }^{37}$ The pseudodielectric function $\langle\epsilon\rangle$ is a common representation of the ellipsometric data $\Psi$ and $\Delta$ via a (ambient-substrate) model $^{37}$ 


$$
\langle\epsilon\rangle=\epsilon_{a}\left([(1-\rho) /(1+\rho)]^{2} \sin ^{2} \Phi_{a}+\cos ^{2} \Phi_{a}\right) \tan ^{2} \Phi_{a} .
$$

The ambient dielectric function $\epsilon_{a}$ is unity. The ellipsometric parameters depend on the photon energy $E$, the sample layer structure, the material dielectric functions, and the angle of incidence $\Phi_{a}$. A model calculation is needed for sample analysis. ${ }^{38}$ We use a two-layer model that includes the Si substrate, the TOFs, the surface roughness, and the ambient. The optical response of TOF is represented by an effective medium approach ${ }^{36}$ in order to distinguish the tantalum oxide dielectric function $\epsilon_{\mathrm{Ta}}$ from the amount of water adsorbed in the TOF micropores. The same effective medium approximation model can simulate the effect of surface roughness for short wavelengths. The IR data did not reveal sensitivity to surface roughness, because scattering becomes inefficient for long wavelengths. The IR and the NIR-VUV experimental data are analyzed independently. Tabulated dielectric function values are used to model the optical response of silicon. ${ }^{39} \mathrm{H}_{2} \mathrm{O}$ optical constants were taken from Palik ${ }^{40}$ for NIR-VUV wavelengths, and from Tiwald et al. ${ }^{41}$ for the IR spectral region. Values for tantalum oxide dielectric function, TOF thickness, film and moisture fractions, and the surface roughness layer thickness remain as unknown parameters. A regression analysis is used to vary the model parameters until the calculated and measured data match as closely as possible. This is done by minimizing the following mean square error function, which is weighted to the estimated experimental errors

$$
\xi^{2}=\frac{1}{2 s-k} \sum_{i=1}^{s}\left[\left(\frac{\Psi_{i}-\Psi_{i}^{c}}{\sigma_{i}^{\sigma}}\right)^{2}+\left(\frac{\Delta_{i}-\Delta_{i}^{c}}{\sigma_{i}^{\Delta}}\right)^{2}\right] .
$$

$S$ denotes the number of measured $\left(\Psi_{i}, \Delta_{i}\right)$ data pairs, $k$ is the number of real-valued fit parameters, and $\Psi_{i}^{c}, \Delta_{i}^{c}$ are the calculated ellipsometric parameters at photon energy $E_{i} .{ }^{42,43}$ The random experimental errors $\left(\sigma_{i}^{\Psi^{\prime}}, \sigma_{i}^{\Delta}\right)$ were appropriately propagated into the error bars on the fit parameters. The error bars also represent finite correlation values between the fit parameters (see Jellison, ${ }^{38}$ see also Ref. 44 ).

Model dielectric functions (MDFs) can greatly reduce the number of free parameters. Simple parametric MDFs such as the Cauchy model for dielectrics, ${ }^{38}$ or the Zollner model for semiconductors native oxides ${ }^{45}$ are often utilized. ${ }^{43,46}$ Separate MDFs are employed in this work for the tantalum oxide IR and NIR-VUV dielectric function spectra, respectively. For the NIR-VUV data a general parametric functional model based on the Kim and Garland approach $^{47-49}$ is used to describe and fit $\epsilon_{\mathrm{Ta}}$ for amorphous tantalum oxide. The mathematical details of this model are discussed elsewhere. ${ }^{50}$ Briefly, the key elements of this model are Kramers-Kronig correct functions with Gaussian broadenings. These functions allow superposition of critical point structures, which are composed of continuous polynomial sections. For this model, 28 internal parameters were used in the fitting process. Some correlation exists among the parameters values. This means that multiple parameter sets exist which all provide the same line shape. However, because we are interested in the final dielectric function rather the final internal model parameter values the fact that mul- tiple parameter sets can produce nearly identical dielectric functions is not a limiting concern. Note that the dielectric function line shape is uniquely determined by the ellipsometric experiment. We have further fitted the line shapes of standard Lorentz oscillator approximations ${ }^{38}$ to the ellipsometric data locally within the spectral region of the fundamental band-to-band transitions. For this procedure we also allowed the structural parameters (surface roughness layer and moisture content in the films) to vary independently (see Sec. IV). We thereby obtained the transition energies and broadening values within the standard Lorentz oscillator critical-point approach for tantalum oxide. ${ }^{38}$

Contributions to $\epsilon_{\mathrm{Ta}}$ due to resonant polar bond vibrations in the IR spectral region $\left(\epsilon_{L}\right)$ are approximated by classical Lorentz oscillator lineshapes

$$
\epsilon_{L}=\epsilon_{\infty}\left[\frac{\omega_{\mathrm{LO}}^{2}-\omega^{2}-i \omega \Gamma_{\mathrm{LO}}}{\omega_{\mathrm{TO}}^{2}-\omega^{2}-i \omega \Gamma_{\mathrm{TO}}}\right],
$$

where $\epsilon_{\infty}$ is the high-frequency dielectric constant, and $\omega_{\mathrm{TO}}$, $\omega_{\mathrm{LO}}$, and $\Gamma_{\mathrm{TO}}, \Gamma_{\mathrm{LO}}$ are the transverse optical (TO) and longitudinal optical (LO) phonon frequencies, and broadening parameters, respectively. ${ }^{51}$

IR-active modes with low polarity can be treated as Lorenztian line shapes with small TO-LO splitting values $\delta \omega^{2}=\omega_{\mathrm{LO}}^{2}-\omega_{\mathrm{TO}}^{2} \ll \omega_{\mathrm{TO}}^{2} .{ }^{52}$ The IR dielectric function can be factored into the lattice contribution $\epsilon_{L}$ and impurity contributions as follows:

$$
\epsilon_{\mathrm{Ta}}=\epsilon_{L} \cdot \prod_{i=1}^{j}\left(1+\frac{i \delta \gamma_{i} \omega-\delta \omega_{i}^{2}}{\omega^{2}+i \gamma_{\mathrm{IM}, i} \omega-\omega_{\mathrm{IM}, i}^{2}}\right),
$$

with the center frequencies $\omega_{\mathrm{IM}, I}$ and broadening parameter $\gamma_{\mathrm{IM}, I}$ of the impurity modes. ${ }^{52}$ The line shape parameter $\delta \gamma_{I}$ accounts for anharmonic coupling effects for spectrally adjacent modes. ${ }^{51,52}$

\section{RESULTS AND DISCUSSION}

XRD spectra of the tantalum oxide thin films did not reveal reflections from tantalum oxide lattice planes. We therefore consider our TOF sample as " $\mathrm{x}$-ray" amorphous. The surface morphology of the TOF samples was studied by AFM (Fig. 1). We found that an increase of deposition temperature and oxygen flux rate improved the surface quality resulting in lower rms values. Figure 1 shows AFM micrographs of the TOF surface morphology for Ta1 (a) and Ta2 (b), which reveal the highest and lowest rms values, respectively. rms values from all samples are summarized in Table III.

The real $\left[\left\langle\epsilon_{1}\right\rangle ;(\mathrm{a})\right]$ and imaginary $\left[\left\langle\epsilon_{2}\right\rangle ;(\mathrm{b})\right]$ parts of the pseudodielectric function $\langle\epsilon\rangle$ from our amorphous TOF samples are shown in Fig. 2 in the spectral region from 0.75 to $8.5 \mathrm{eV}$. The graphs are shifted for convenience. The symbols refer to the experimental NIR-VUV-SE data, and the solid lines correspond to the best-fit calculation. Near and below the band-gap energy $E_{g}$ (see also Table III) the pseudodielectric function spectra are dominated by multiple reflections within the film interfaces, whereas differences in film thickness result in different interference patterns. Note that the best-fit calculation accurately matches the experi- 


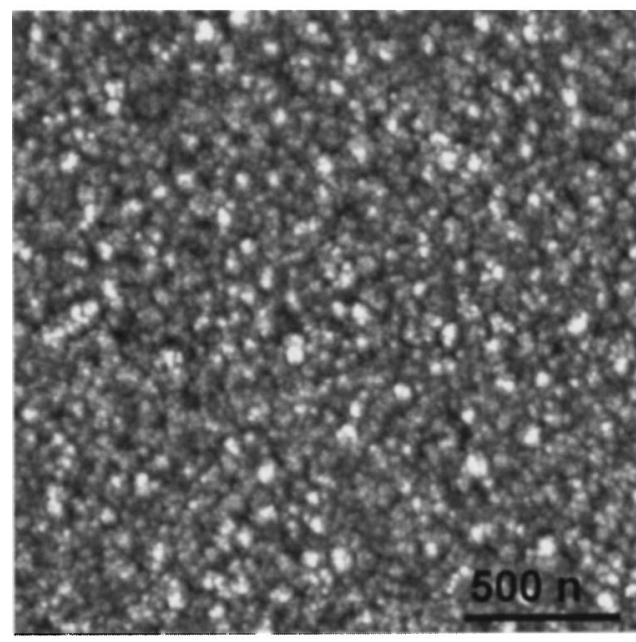

$25 \mathrm{~nm}$

(a)

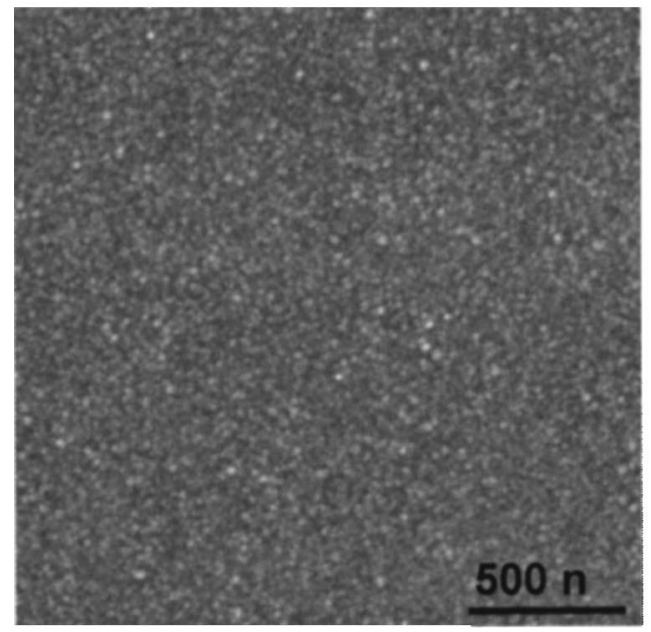

$25 \mathrm{~nm}$

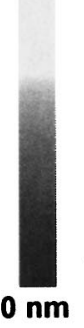

(b)

FIG. 1. AFM images from the surface morphology of TOF samples Ta1 (a) and $\mathrm{Ta} 2$ (b). The rms values of 2.76 and $1.25 \mathrm{~nm}$ for Ta1 and Ta2, respectively, were considered as a measure of the surface roughness.

mental data, especially in the near-band-gap region. The experimental data were analyzed using a two-layer model. An effective medium approach was used to model the optical properties of the TOF and the surface roughness layer. For TOF the effective dielectric function $\epsilon$ follows from

$$
0=f_{m} \frac{\epsilon_{m}-\epsilon}{\epsilon_{m}+2 \epsilon}+\left(1-f_{m}\right) \frac{\epsilon_{\mathrm{Ta}}-\epsilon}{\epsilon_{\mathrm{Ta}}+2 \epsilon},
$$

where $\epsilon_{m}, \epsilon_{\mathrm{Ta}}$, and $f_{m}, f_{\mathrm{Ta}}=\left(1-f_{m}\right)$ are the dielectric functions and volume fractions of $\mathrm{H}_{2} \mathrm{O}(m)$ and tantalum ox-
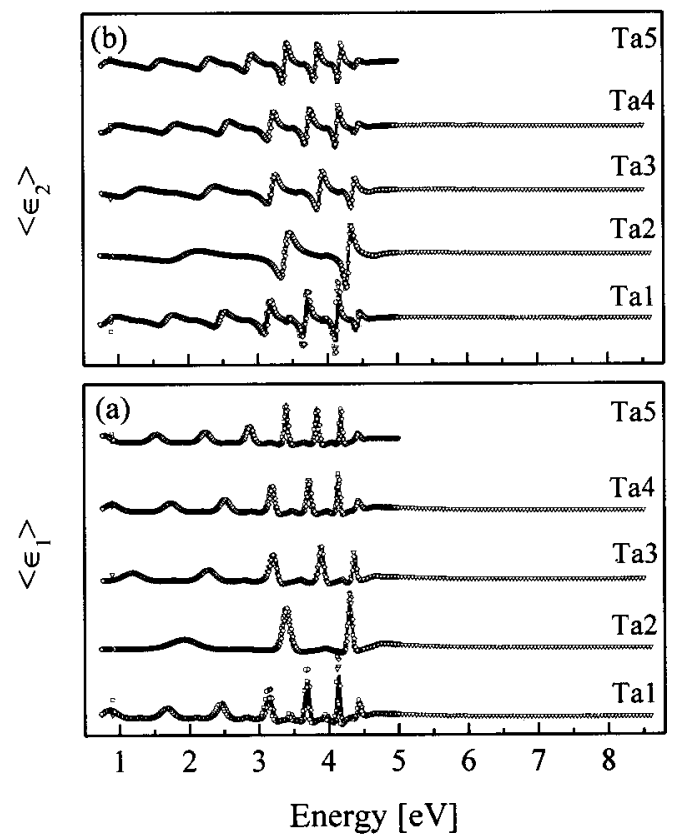

FIG. 2. Pseudodielectric function $\langle\epsilon\rangle=\left\langle\epsilon_{1}\right\rangle+i\left\langle\epsilon_{2}\right\rangle$ of amorphous tantalum oxide thin films. The solid lines correspond to the best fit of the experimental data [symbols, $\Phi_{a}=65^{\circ}$ (squares), $70^{\circ}$ (diamonds), $75^{\circ}$ (up triangles)]. Parameters obtained from regression analysis are summarized in Table III. The experimental data reveal the onset of absorption due to the band gap at $4.5 \mathrm{eV}$. Below the band gap the material is transparent. Above the band gap the material is absorbing with no higher energy transitions observed. Experimental VUV data for Ta5 are not available. Graphs are shifted for convenience. $\left[\left\langle\epsilon_{1}\right\rangle:(a),\left\langle\epsilon_{2}\right\rangle:(b)\right]$.

ide (Ta), respectively. The same formalism can be used to effectively model surface roughness assuming an average void fraction $(v)$ of $50 \%$ within a virtual top layer with thickness $d_{\text {rough }}\left(\epsilon_{v}=1\right)$. The dielectric function spectra $\epsilon_{\mathrm{Ta}}$ were obtained using a generalized parametric semiconductor dielectric function model. ${ }^{47-50}$ This model possesses high flexibility with respect to arbitrary but Kramers-Kronig consistent line shapes of the real $\left(\epsilon_{1}\right)$ and imaginary $\left(\epsilon_{2}\right)$ parts of $\epsilon_{\mathrm{Ta}}$.

Dielectric function line shape for amorphous tantalum oxide, TOF thickness, percentage of moisture fraction and thickness of the surface roughness layer were then obtained from the regression analysis of the experimental data (see Table III). Table III also contains the index of refraction $n$ at $\lambda=550 \mathrm{~nm}(E=2.48 \mathrm{eV})$. Except for Ta3 we obtain a highly consistent index value of approximately $2.13 \ldots 2.16$ from all samples despite their different microstructures. The surface

TABLE III. Sample structure parameters and band-gap energies obtained from NIR-VUV-SE data and AFM analysis.

\begin{tabular}{cccccccc}
\hline \hline Sample & $\begin{array}{c}t \\
{[\mathrm{~nm}]}\end{array}$ & $\begin{array}{c}E_{g 1} \\
{[\mathrm{eV}]}\end{array}$ & $\begin{array}{c}E_{g 2} \\
{[\mathrm{eV}]}\end{array}$ & $\begin{array}{c}n \\
\lambda=550 \mathrm{~nm}\end{array}$ & $\begin{array}{c}f_{m} \\
{[\%]}\end{array}$ & $\begin{array}{c}t_{\text {rough }}[\mathrm{nm}] \\
(\mathrm{SE})\end{array}$ & $\begin{array}{c}\text { rms [nm }] \\
(\mathrm{AFM})\end{array}$ \\
\hline $\mathrm{Ta} 1$ & $426 \pm 5$ & $4.65 \pm 0.05$ & $5.43 \pm 0.06$ & 2.13 & $16 \pm 2$ & $9.1 \pm 0.2$ & 3 \\
$\mathrm{Ta} 2$ & $175 \pm 3$ & $4.89 \pm 0.08$ & $5.26 \pm 0.09$ & 2.14 & $2 \pm 1$ & $4.4 \pm 0.1$ & 1 \\
$\mathrm{Ta} 3$ & $316 \pm 5$ & $4.68 \pm 0.03$ & $5.45 \pm 0.04$ & 2.04 & $2 \pm 1$ & $8.1 \pm 0.1$ & 2 \\
Ta4 & $394 \pm 5$ & $4.73 \pm 0.03$ & $5.51 \pm 0.04$ & 2.16 & $4 \pm 1$ & $6.3 \pm 0.1$ & 2 \\
Ta5 & $465 \pm 5$ & $4.63 \pm 0.03$ & & 2.16 & $2 \pm 1$ & $5.8 \pm 0.1$ & 2 \\
\hline \hline
\end{tabular}



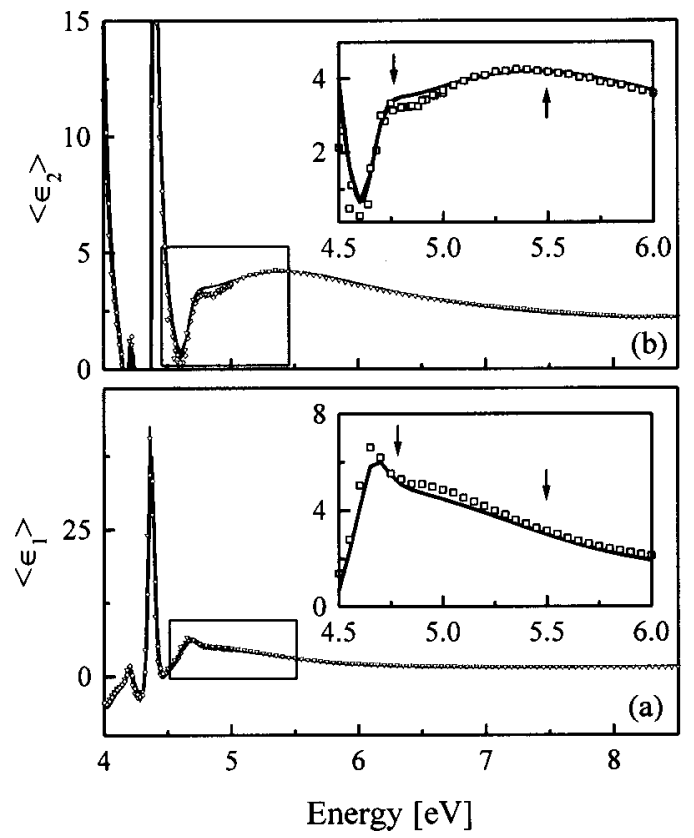

FIG. 3. Experimental (symbols) and generated (solid line) pseudodielectric function $\langle\epsilon\rangle$ for Ta3 in the UV-VUV spectral region $\left[\left\langle\epsilon_{1}\right\rangle:(a),\left\langle\epsilon_{2}\right\rangle\right.$ : (b) of $\langle\epsilon\rangle]$. The insets enlarge the fundamental band-gap region. Two transitions were found as indicated by arrows. The solid lines within the insets are the best-fit Lorentz oscillator line shapes obtained from analysis of the experimental data for $E=4.5 \ldots 6 \mathrm{eV}$.

roughness layer thickness compares well with the percentage of void fraction and the rms value obtained by AFM (Table III). The TOF with the largest (smallest) surface roughness layer thickness also shows the largest (smallest) rms value, respectively. A similar tendency is observed for the rms values and the percentage of void fraction.

Figure 3 shows a magnification of the real (a) and imaginary parts (b) of $\langle\epsilon\rangle$ from Ta3 for photon energies from 4 to $8.5 \mathrm{eV}$. Absorption within the amorphous TOF occurs due to the onset of band-to-band transitions in this spectral region. The insets in Figs. 3(a) and 3(b) further enlarge the band-gap region from 4.5 to $6.0 \mathrm{eV}$. The vertical arrows indicate the two band-to-band transition energies, which were identified from line shape analysis of the amorphous tantalum oxide dielectric function $\epsilon_{\mathrm{Ta}}$. Spectra for $\epsilon_{\mathrm{Ta}}$ were extracted during analysis of the ellipsometry data using a generalized parametric dielectric function model. ${ }^{50}$ The symbols in Fig. 3 refer to the experimental data points, and the solid lines are the best-fit calculations employing $\epsilon_{\mathrm{Ta}}$ (see also Fig. 8), and TOF thickness and void fractions and surface roughness layer thickness as discussed above (see Table III). Note the excellent agreement between experimental and best-fit model data. The energies of the band-to-band transitions were obtained by locally fitting Lorentzian line shapes to the experimental ellipsometry spectra from 4.2 to $6 \mathrm{eV}$. The solid lines within the insets of Figs. 3(a) and 3(b) refer to the best-fit line shape using two Lorentz oscillators. In this spectral region the Lorentz line shapes are almost identical to that obtained by the generalized parametric dielectric function model [solid lines in Figs. 3(a) and 3(b)]. The center frequencies of the Lorentz line shapes are treated here as characteristic band-to-band transition energies for our TOF films.

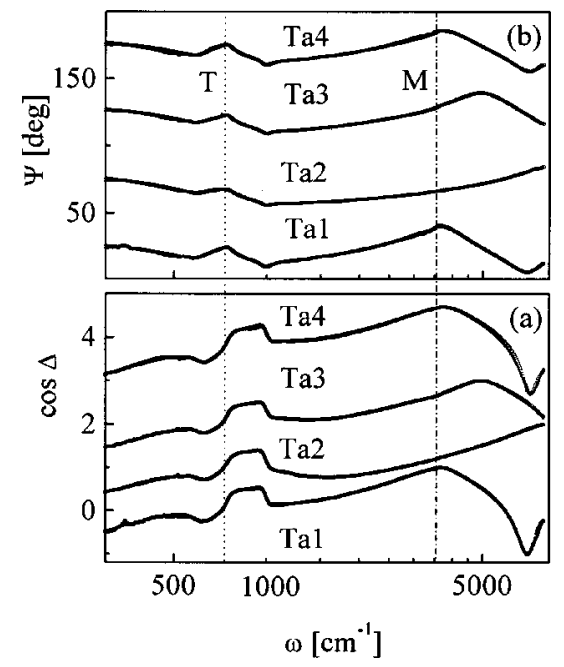

FIG. 4. Experimental (symbols) and generated IR ellipsometry data (solid lines) obtained from sample $\mathrm{Ta} 1$ to $\mathrm{Ta} 4$ in the wave number region from 300 to $8000 \mathrm{~cm}^{-1}(0.03-1 \mathrm{eV})\left[(\mathrm{a}): \Psi ;(\mathrm{b}): \cos (\Delta) ; \Phi_{a}=70^{\circ}\right]$. The spectra for $\mathrm{Ta} 1, \mathrm{Ta} 3$, and Ta4 show near to the thickness interference a broad absorption near $3600 \mathrm{~cm}^{-1}$ due to water incorporation as indicated by dasheddotted line $(M)$. The dotted line $(T)$ refers to the amorphous tantalum oxide phonon resonance. Graphs are shifted for convenience, and " $\Delta$ " spectra are transformed into the " $\cos \Delta$ " presentation to keep graphs bound between -1 and 1 .

The structural parameters $f_{m}$ and $t_{\text {rough }}$ were also allowed to vary during the local Lorentz line shape analysis, but found to be stable inside their error range (see Table III) with respect to the results obtained using the general parameter function set and the NIR-VUV wavelength range fit. $E_{g 1}$ and $E_{g 2}$ vary slightly for different samples, and the results are summarized in Table III. Note that no experimental VUV data were available for sample Ta5 because the TOF peeled off the substrate soon after deposition, which prevented determination of $E_{g 2}$ for Ta5.

Figures 4(a)and 4(b) present experimental (symbols) and generated (solid lines) IR ellipsometry spectra from samples Ta1 to Ta4 for wave numbers from $\omega=300$ to $8000 \mathrm{~cm}^{-1}$. $[0.03-1 \mathrm{eV} ; \Psi$ : (a), $\Delta$ : (b); spectra are shifted for convenience.] Surface roughness, when affecting UV-VUV ellipsometry data to some extent only, is not significant for long wavelength investigations. The experimental data were therefore analyzed employing the same model as used for the NIR-VUV data except for the surface roughness layer. The generated data of Figs. 4(a) and 4(b) correspond to the bestfit model calculation of $\epsilon_{\mathrm{Ta}}$ using Lorentzian line shapes. The amorphous tantalum oxide dielectric function parameters are $\omega_{\mathrm{TOa}}, \omega_{\mathrm{LOa}}, \Gamma_{\mathrm{TOa}}, \Gamma_{\mathrm{LOa}}, \epsilon_{\infty}$, frequencies $\left(\omega_{\mathrm{IMP}}\right)$ and broadening values $\left(\Gamma_{\text {IMP }}\right)$ of several impurity modes, and a long-tail contribution from polar resonance absorption with center frequency near zero $\left(\omega_{\mathrm{TO} 0}=0\right)$. TOF thickness and percentage of void fraction are further model parameters. The best-fit results are summarized in Table IV. Similar to $n$ in the NIR-VUV spectral region the TOF samples, except Ta3, show a highly consistent $\boldsymbol{\epsilon}_{\infty}$ value, in spite of different microporosities. Amorphous tantalum oxide phonon resonances (dotted line in Fig. 4) and impurity modes are located in the wave number region between $\omega=600$ and $1300 \mathrm{~cm}^{-1}$. 
TABLE IV. Phonon mode frequencies and sample structure parameters obtained from IR-SE data analysis.

\begin{tabular}{|c|c|c|c|c|c|c|c|c|c|c|}
\hline & $\mathrm{TO}_{a}\left[\mathrm{~cm}^{-1}\right]$ & $\mathrm{LO}_{a}\left[\mathrm{~cm}^{-1}\right]$ & $\operatorname{IM} 1\left[\mathrm{~cm}^{-1}\right]$ & $\mathrm{IM} 2\left[\mathrm{~cm}^{-1}\right]$ & $\mathrm{IM} 3\left[\mathrm{~cm}^{-1}\right]$ & $\mathrm{IM} 4\left[\mathrm{~cm}^{-1}\right]$ & $\epsilon_{\infty}$ & $\begin{array}{c}\mathrm{LO}_{0} \\
{\left[\mathrm{~cm}^{-1}\right]}\end{array}$ & $t[\mathrm{~nm}]$ & $f_{m}[\%]$ \\
\hline Ta1 & $647 \pm 3$ & $778 \pm 2$ & $734 \pm 2$ & $977 \pm 2$ & & & $4.2 \pm 0.1$ & $587 \pm 9$ & $422 \pm 1$ & $18 \pm 1$ \\
\hline Тa2 & $631 \pm 3$ & $794 \pm 2$ & $734 \pm 3$ & $980 \pm 2$ & & & $4.2 \pm 0.1$ & $504 \pm 14$ & $165 \pm 1$ & $0 \pm 1$ \\
\hline Ta3 & $631 \pm 2$ & $787 \pm 3$ & $733 \pm 2$ & $976 \pm 2$ & & & $3.8 \pm 0.1$ & $562 \pm 7$ & $312 \pm 1$ & $6 \pm 1$ \\
\hline Ta4 & $639 \pm 2$ & $787 \pm 2$ & $734 \pm 2$ & $979 \pm 2$ & & & $4.3 \pm 0.1$ & $535 \pm 6$ & $394 \pm 1$ & $10 \pm 1$ \\
\hline Ta5 & $647 \pm 2$ & $778 \pm 2$ & $735 \pm 2$ & $1048 \pm 2$ & $1253 \pm 1$ & $1306 \pm 1$ & ...a) & $503 \pm 11$ & ...a) & ...a) \\
\hline
\end{tabular}

a) TOF thickness, $\boldsymbol{\epsilon}_{\infty}$ and moisture fraction $f_{m}$ parameter are not given for Ta5. Although it was fitted for those parameters, they did not characterize real sample properties, because the sample was peeled off the substrate.

Additional small resonance structures are caused by moisture, and were detected within samples Ta1, Ta3, and Ta4 near $\omega=3600 \mathrm{~cm}^{-1}$ (dash-dotted line in Fig. 4). The features above $1100 \mathrm{~cm}^{-1}$ with a long period are due to thickness interference effects within the TOF samples. The phonon resonance parameters $\omega_{\mathrm{TOa}}$, and $\omega_{\mathrm{LO}}$ do not change significantly for samples grown under different conditions. Deposition parameters only influence the growth rate (film thickness) and therefore the porosity and the TOF moisture incorporation. Note that TOF thickness obtained from IR and NIR-VUV data analysis are the same within the experimental error bars. The IR void fraction reveals a similar trend with deposition parameters as that obtained from the NIRVUV data. Note also that the IR-SE data were measured after taking the NIR-UV and UV-VUV data, and moisture absorption may have increased between experiments.

Figures 5(a) and 5(b) show details of experimental (open cycles) and generated (solid lines) $\Psi$ and $\Delta$ spectra from sample Ta1 for wave numbers from $\omega=350$ to $2250 \mathrm{~cm}^{-1}$. The model function for the generated data include one Lorentzian absorption line shape at $\omega_{\mathrm{TO}}=648 \mathrm{~cm}^{-1}$ with $\omega_{\mathrm{LOa}}=734 \mathrm{~cm}^{-1}, \Gamma_{\mathrm{TOa}}, \Gamma_{\mathrm{LOa}}$, and $\epsilon_{\infty}=4.2$. Two impurity modes were further included at $\omega_{\mathrm{IM} 1}=778 \mathrm{~cm}^{-1}$ and $\omega_{\mathrm{IM} 2}$ $=977 \mathrm{~cm}^{-1}$. A long-tail background absorption was neces-

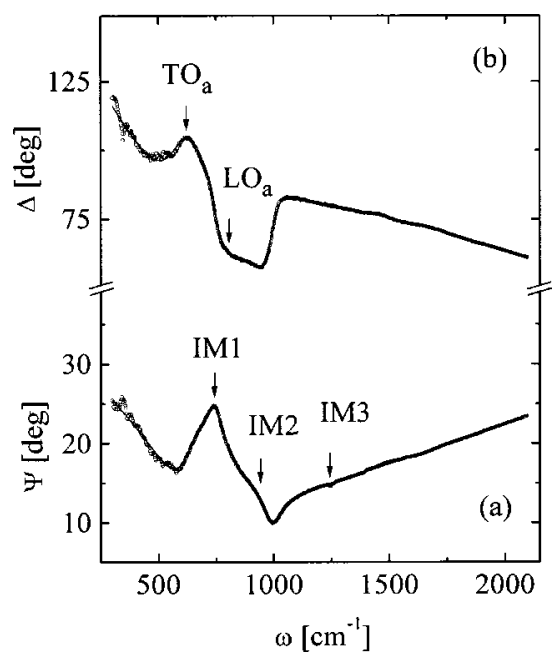

FIG. 5. Experimental (circles) and generated IR ellipsometry data (solid line) obtained from sample Ta1. Arrows indicate the spectral position of the amorphous tantalum oxide phonon modes at $\omega_{\mathrm{TO}}=648 \mathrm{~cm}^{-1}\left(\mathrm{TO}_{a}\right)$, and $\omega_{\mathrm{LO}}=778 \mathrm{~cm}^{-1}\left(\mathrm{LO}_{a}\right)$, and the spectral positions of the impurity modes at $\omega_{\mathrm{IM} 1}=734 \mathrm{~cm}^{-1}$, and $\omega_{\mathrm{IM} 2}=977 \mathrm{~cm}^{-1}$. Note that $\omega_{\mathrm{IM} 3} \sim 1251 \mathrm{~cm}^{-1}$ was not fitted during data analysis. sary to account for the increase in $\operatorname{Im}\left\{\epsilon_{\mathrm{Ta}}\right\}$ and the decrease in $\operatorname{Re}\left\{\epsilon_{\mathrm{Ta}}\right\}$ for long wavelengths (See also Figs. 7 and 8). This absorption was modeled by a Lorentzian line shape with center energy set to infinite wavelength $\left(\omega_{\mathrm{TO} 0}=0 \mathrm{~cm}^{-1}\right)$. To reveal the origin of this long-tail contribution ellipsometry investigations should be performed at much longer wavelengths than at those measured here. Tantalum oxide is known as a material with a large static dielectric constant. Therefore $\operatorname{Im}\left\{\epsilon_{\mathrm{Ta}}\right\}$ should be zero and $\operatorname{Re}\left\{\epsilon_{\mathrm{Ta}}\right\}$ should be large and positive at zero photon energy. This can only be achieved in our model if $\omega_{\mathrm{TO}}>0 \mathrm{~cm}^{-1}$. This would indicate a strong but polar optical mode at long wavelengths in amorphous tantalum oxide. On the other hand, free carriers within the TOF samples could screen the polar lattice bond resonances, and the long-tail contribution would then be due to IR-optical free-carrier effects. Up to this point we are unable to differentiate between both effects, as the IR investigations were limited to $0.03 \mathrm{eV}$. The $\mathrm{LO}_{0}$ frequencies (in Table IV) and the broadening parameters may be regarded here as necessary parameters to account for the low-frequency behavior of our IR data.

The spectral position of another weak impurity-like mode $\left(\omega_{\text {IM3 }}\right)$ is indicated in Fig. 5(a). However, the small contributions due to $\omega_{\mathrm{IM} 3}$ were not considered during analysis of samples Ta1-Ta4. Figures 6(a) and 6(b) present details of experimental (open cycles) and generated (solid lines) $\Psi$ and $\Delta$ spectra from sample Ta5 for wave numbers from $\omega$ $=350$ to $2250 \mathrm{~cm}^{-1}$. This sample had already been peeled off the substrate before the IR data were measured, and it was necessary to modify the model assumptions for ellipsometry data analysis. In addition, absorption features due to native silicon oxide $\left(\mathrm{TO}_{\mathrm{SiO} 2}\right)$, two additional impurity-like modes $\left(\omega_{\mathrm{IM} 3}, \omega_{\mathrm{IM} 4}\right)$ were necessary to model the strong changes in $\Psi$ and $\Delta$ between 1000 and $1500 \mathrm{~cm}^{-1}$. The resulting parameters for $\omega_{\mathrm{TOa}}, \omega_{\mathrm{LOa}}, \epsilon_{\infty}, \omega_{\mathrm{LO} 0}$, and the four impurity modes are given in Table IV as well. The origins for the impurity-like modes are still unknown. At least we tentatively assign $\omega_{\mathrm{IM} 3}$ and $\omega_{\mathrm{IM} 4}$ to $\mathrm{Ta}-\mathrm{OH}$ or $\mathrm{Ta}-\mathrm{H}$ bonding absorptions because reactions of hydrogen and hydroxyl with tantalum may occur at dangling bonds in the amorphous tantalum oxide network. The increased intensity of $\omega_{\text {IM } 3}$ and $\omega_{\text {IM4 } 4}$ in sample Ta5 can be explained by the increased reaction surface after the film has peeled off the substrate.

The best-fit amorphous tantalum oxide optical function spectra for sample $\mathrm{Ta} 2$ is shown as an example in Figs. 7 and 8 in terms of the real (Fig. 7(a): $\epsilon_{1}$; Fig. 8(a): $n$ ) and imagi- 


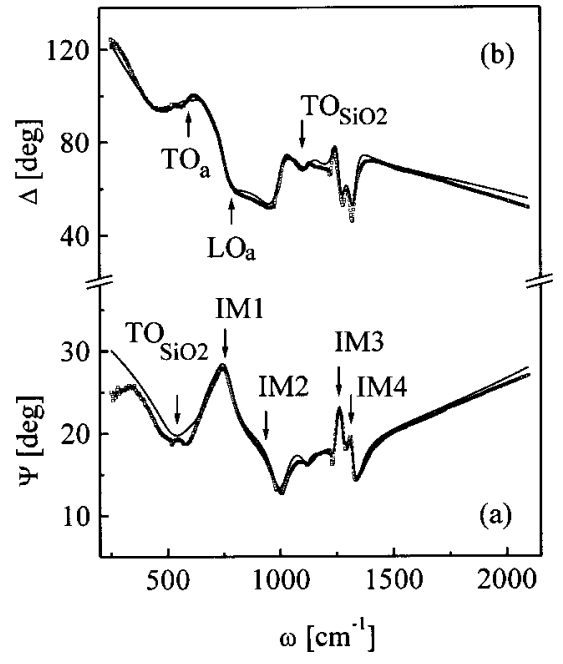

FIG. 6. Experimental (circles) and generated IR ellipsometry data (solid line) obtained from sample Ta5. Contribution from the native $\mathrm{SiO}_{2}$ layer at $\omega_{\mathrm{SiO} 2}=470 \mathrm{~cm}^{-1}$, and $\omega_{\mathrm{SiO} 2}=1070 \mathrm{~cm}^{-1}$ were found, because the film had many cracks and partly peeled off from the substrate. Arrows indicate the spectral position of the amorphous tantalum oxide phonon modes, and the spectral positions of four impurity modes observed here.

nary (Fig. 7(b): $\epsilon_{2}$; Fig. 8(b): $k$ ) parts of the dielectric function or optical constants, respectively. Significant model parameters are listed in Tables III and IV. Below the band gap the TOF sample is transparent. The region of transparency is limited in the long-wavelength range by resonant bonding absorption, and in the short-wavelength region by the onset of absorption due to electronic band-to-band transitions. A rather sharp onset of the band-to-band absorption is observed in all of our TOF samples. As discussed above, the increase (decrease) in $\epsilon_{2}\left(\epsilon_{1}\right)$ below the tantalum oxide phonon reso-

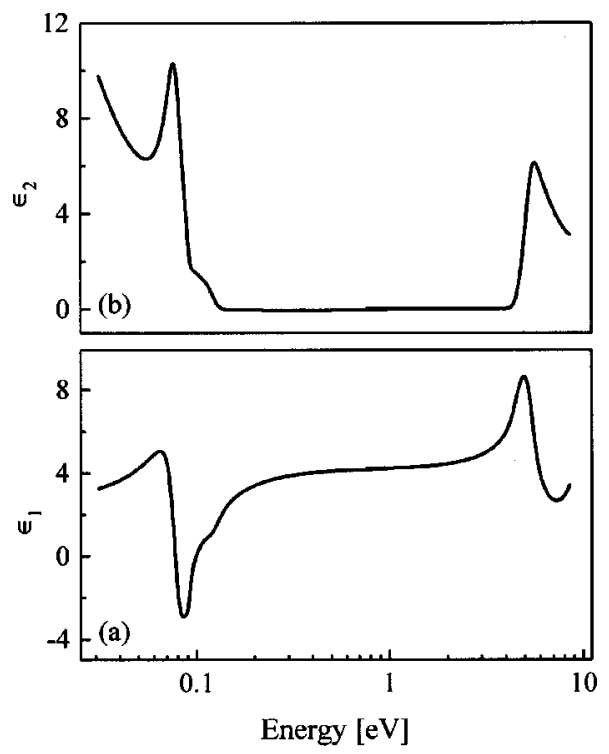

FIG. 7. Dielectric function $\epsilon=\epsilon_{1}+i \epsilon_{2}$ of amorphous tantalum oxide thin films, extracted from line shape analysis of experimental ellipsometry data, [(a): $\left.\epsilon_{1},(b): \epsilon_{2}\right]$. Note the sharp onset of absorption caused by the band gap. The imaginary part $\epsilon_{2}$ between the phonon region and the onset of electronic band-to-band transition is too small to be measured by spectroscopic ellipsometry and at least less than the experimental error bar on each $\epsilon_{2}$ value $\left(\epsilon_{2}<0.01\right)$.

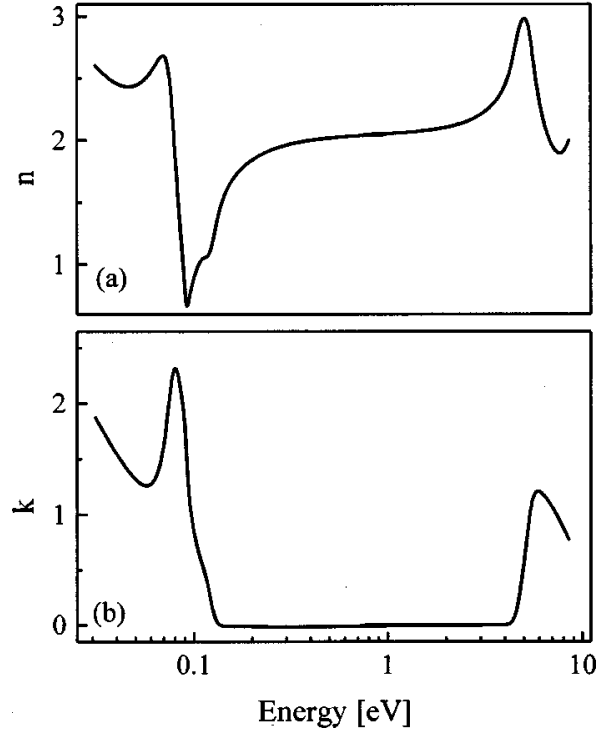

FIG. 8. Same as Fig. 7 for $N=n+i k$, [(a): $n,(\mathrm{~b}): k]$.

nance near $\sim 0.09 \mathrm{eV}$ is due to a strong polar absorption mechanisms with center wavelength much longer than used for our investigations here. Note that the optical function spectra extracted from the IR and the NIR-VUV data perfectly match within the experimental error bars.

\section{CONCLUSIONS}

The dielectric function $\epsilon=\epsilon_{1}+\mathrm{i} \epsilon_{2}$ and the critical point parameters, such as the band-gap energy, and the spectral position of transverse and longitudinal optical phonon are reported for amorphous tantalum oxide thin films in the photon energy range from $E=0.03$ to $8.5 \mathrm{eV}$. The samples were grown with different oxygen flux, deposition temperatures, and deposition pressures. XRD measurements revealed their amorphous structure. AFM investigations provided a measure of sample surface roughness.

The NIR-VUV-SE data were analyzed using a twolayer model, one layer for the tantalum oxide optical properties including the thin film moisture fraction employing an effective medium approach, and a surface roughness layer. Two band-gap energies were found at $\left(E_{g 1} \sim 4.7 \mathrm{eV}\right)$, and at $E_{g 2} \sim 5.4 \mathrm{eV}$ for all samples.

The IR-SE spectra revealed the transverse and longitudinal optical phonon frequencies for amorphous tantalum oxide at $\omega_{\mathrm{TO}}=631 \mathrm{~cm}^{-1}$ and $\omega_{\mathrm{LO}}=787 \mathrm{~cm}^{-1}$, respectively. Additional weak absorptions were treated as impurity modes, and found at $\omega_{\mathrm{IM} 1}=733 \mathrm{~cm}^{-1}$ and $\omega_{\mathrm{IM} 2}=976 \mathrm{~cm}^{-1}$. Two further impurity modes were observed at $\omega_{\mathrm{IM} 3}=1253 \mathrm{~cm}^{-1}$ and $\omega_{\mathrm{IM} 4}=1306 \mathrm{~cm}^{-1}$ within a sample where the tantalum oxide film peeled off from the substrate.

Refractive index $n$ fit in NIR-VUV, and high-frequency dielectric constant $\epsilon_{\infty}$ fit in the IR, are consistent for the tantalum oxide films, despite different sample microporosities.

Results from ellipsometry analysis for film thickness, surface roughness, and volume fraction of moisture incorpo- 
ration are consistent between models for IR and NIR-VUV data analysis, as well as with AFM results.

\section{ACKNOWLEDGMENTS}

The authors acknowledge D. W. Thompson at the University of Nebraska, Lincoln for technical guidance. They further thank C. M. Herzinger at J. A. Woollam Co. Lincoln, Nebraska for valuable comments and discussions during the generalized parametric model analysis. The work was supported by BMDO Contract No. DSAG60-98-C-0054, NASA Glenn Research Center Grant No. NAG3-2219, and NASA Epscor Grant No. NCC5-169.

${ }^{1}$ A. J. Waldorf, J. A. Dobrowolski, B. T. Sullivan, and L. M. Plante, Appl. Opt. 32, 5583 (1993).

${ }^{2}$ F. Rubio, J. M. Albella, J. Denis, and J. M. Martinez-Duart, J. Vac. Sci. Technol. 21, 1043 (1982).

${ }^{3}$ W. M. Paulson, F. S. Hickernell, and R. L. Davis, J. Vac. Sci. Technol. 16, 307 (1979).

${ }^{4}$ I. Proqueras, J. Marti, and E. Bertran, Thin Solid Films 343, 449 (1999).

${ }^{5}$ M. Kitao, H. Akram, K. Urabe, and S. Yamada, J. Electron. Mater. 21, 419 (1992)

${ }^{6}$ P. C. Joshi and M. W. Cole, J. Appl. Phys. 86, 871 (1999).

${ }^{7}$ A. Pignolet, G. M. Rao, and S. B. Krupanidhi, Thin Solid Films 258, 230 (1995).

${ }^{8}$ S. Ezhilvalavan and T. Y. Tseng, J. Am. Ceram. Soc. 82, 600 (1999).

${ }^{9}$ S. W. Park and H. B. Im, Thin Solid Films 207, 258 (1992).

${ }^{10}$ V. Mikhaelashvili, Y. Betzer, I. Prudnikov, M. Orenstein, D. Ritter, and G. Eisenstein, J. Appl. Phys. 84, 6747 (1998).

${ }^{11}$ F. C. Chiu, J. J. Wang, J. Y. Lee, and S. C. Wu, J. Appl. Phys. 81, 6911 (1997).

${ }^{12}$ S. O. Kim, J. S. Byun, and H. J. Kim, Thin Solid Films 206, 102 (1991).

${ }^{13}$ S. W. Park, Y. K. Baek, J. Y. Lee, C. O. Park, and H. B. Im, J. Electron. Mater. 21, 635 (1992).

${ }^{14}$ C. Chaneliere, S. Four, J. L. Autran, R. A. B. Devine, and N. P. Sandler, J. Appl. Phys. 83, 4823 (1998).

${ }^{15}$ B. K. Moon, C. Isobe, and J. Aoyama, J. Appl. Phys. 85, 1731 (1999).

${ }^{16}$ I. Kim, J. S. Kim, O. S. Kwon, S. T. Ahn, J. S. Chun, and W. J. Lee, J. Electron. Mater. 24, 1435 (1995).

${ }^{17}$ R. A. B. Devine, L. Vallier, J. L. Autran, P. Paillet, and J. L. Leray, Appl. Phys. Lett. 68, 1775 (1996).

${ }^{18}$ M. Schubert, B. Rheinländer, E. Franke, H. Neumann, J. Hahn, M. Röder, and F. Richter, Appl. Phys. Lett. 70, 1819 (1997).

${ }^{19}$ M. Schubert, B. Rheinländer, E. Franke, H. Neumann, T. E. Tiwald, J. A. Woollam, J. Hahn, and F. Richter, Phys. Rev. B 56, 13306 (1997).

${ }^{20}$ E. Franke, M. Schubert, H. Neumann, T. E. Tiwald, D. W. Thompson, J. A. Woollam, J. Hahn, and F. Richter, J. Appl. Phys. 82, 2906 (1997).

${ }^{21}$ E. Franke, M. Schubert, J.-D. Hecht, H. Neumann, T. E. Tiwald, H. Yao, J. A. Woollam, and J. Hahn, J. Appl. Phys. 84, 526 (1998).
${ }^{22}$ M. Cevro and G. Carter, Opt. Eng. 34, 603 (1995).

${ }^{23}$ H. Demiryont, J. R. Sites, and K. Gelb, Appl. Opt. 24, 490 (1985).

${ }^{24}$ W. B. Westwood, R. J. Boynton, and S. J. Ingrey, J. Vac. Sci. Technol. 11, 381 (1974).

${ }^{25}$ X. H. Pan, S. L. Qiu, M. I. Florit, M. L. Shek, and M. Strongin, Phys. Rev. B 35, 3740 (1987).

${ }^{26}$ D. J. Werder and R. R. Kola, Thin Solid Films 323, 6 (1998).

${ }^{27}$ K. Gürtler, K. Bange, W. Wagner, F. Rauch, and H. Hantsche, Thin Solid Films 175, 185 (1989).

${ }^{28}$ Y. Nishimura, A. Shinkawa, H. Ujita, M. Tsuji, and M. Nakamura, Appl. Surf. Sci. 136, 22 (1998).

${ }^{29}$ Z. W. Fu, L. Y. Chen, and Q. Z. Qin, Thin Solid Films 340, 164 (1999).

${ }^{30}$ G. B. Alers, R. M. Fleming, Y. H. Wong, B. Dennis, A. Pinczuk, G. Redinbo, R. Urdahl, E. Ong, and Z. Hasan, Appl. Phys. Lett. 72, 1308 (1998).

${ }^{31}$ J. Y. Zhang, B. Lim, and I. W. Boyd, Thin Solid Films 336, 340 (1998).

${ }^{32}$ J. Y. Zhang, B. Lim, and I. W. Boyd, Appl. Phys. Lett. 73, 2299 (1998).

${ }^{33}$ K. Kukli, J. Aarik, A. Aidla, O. Kohan, T. Uustare, and V. Sammelselg, Thin Solid Films 260, 135 (1995).

${ }^{34}$ D. Laviale, J. C. Oberlin, and R. A. B. Devine, Appl. Phys. Lett. 65, 2021 (1994).

${ }^{35}$ I. Hodgkinson, Q. H. Wu, and J. Hazel, Appl. Opt. 37, 2653 (1998).

${ }^{36}$ D. Aspnes and J. B. Theeten, Phys. Rev. B 20, 3292 (1979).

${ }^{37}$ R. M. A. Azzam, and N. M. Bashara, Ellipsometry and Polarized Light (North-Holland, Amsterdam, 1977).

${ }^{38}$ G. E. Jellison, Thin Solid Films 313-314, 33 (1998).

${ }^{39}$ C. M. Herzinger, B. Johs, W. A. McGahan, J. A. Woollam, and W. Paulson, J. Appl. Phys. 83, 3323 (1998).

${ }^{40}$ E. D. Palik, Handbook of Optical Constant of Solids (Academic, New York, 1998)

${ }^{41}$ T. E. Tiwald, D. W. Thompson, J. A. Woollam, and S. V. Pepper, Thin Solid Films 313-314, 718 (1998).

${ }^{42}$ C. M. Herzinger, P. G. Snyder, B. Johs, and J. A. Woollam, J. Appl. Phys. 77, 1715 (1995)

${ }^{43}$ C. M. Herzinger, H. Yao, P. G. Snyder, F. G. Celii, Y. C. Kao, B. Johs, and J. A. Woollam, J. Appl. Phys. 77, 4677 (1995).

${ }^{44}$ Guide to Use WVASE 32 (J. A. Woollam Co., Inc., Lincoln, NE, 1995)

${ }^{45}$ S. Zollner, Appl. Phys. Lett. 63, 2523 (1993).

${ }^{46}$ M. Schubert, J. A. Woollam, G. Leibiger, B. Rheinländer, I. Pietzonka, T. Sass, and V. Gottschalch, J. Appl. Phys. 86, 2025 (1999).

${ }^{47}$ C. C. Kim, J. W. Garland, H. Abad, and P. M. Raccah, Phys. Rev. B 45, 11749 (1992)

${ }^{48}$ C. C. Kim, J. W. Garland, and P. M. Raccah, Phys. Rev. B 47, 1876 (1993).

${ }^{49}$ J. W. Garland, C. Kim, H. Abad, and P. M. Raccah, Phys. Rev. B 41, 7602 (1990).

${ }^{50}$ C. M. Herzinger and B. D. Johs, US Patent No. 5796983 (1998).

${ }^{51}$ M. Schubert, T. E. Tiwald, and C. M. Herzinger, Phys. Rev. B 61, 8187 (2000).

52 A. Kasic, M. Schubert, T. E. Tiwald, S. Einfeldt, and D. Hommel, Phys. Rev. B 62, 7365 (2000). 